\title{
ULTRASONOGRAFIA DEL MANGUITO DE LOS ROTADORES DEL HOMBRO: EVALUACION POST-QUIRURGICA
}

\author{
Drs. Paulina Yánez S, M. Eugenia Lúcia C, Andrea Glasinovic P, Sergio Montenegro M.
}

Clínica Dávila - Clínica Santa María.

\begin{abstract}
Forty (40) patients operated from rotator cuff tears were studied using ultrasound (US) study to investigate features of the operated tendon and the stability of the scar. We found a $27.7 \%$ of recurrent tears (finding previously reported). Correlation with arthroscopic exploration is also demonstrated showing good sensitivity ultrasound examination. We consider ultrasonography as an alternative for the post surgical evaluation of the rotator cuff.

Key words: Ultrasound, Rotator cuff.
\end{abstract}

Resumen: Se estudiaron con ultrasonografía 40 pacientes operados de manguito rotador, en forma dinámica para pesquisar las características del tendón operado y la estabilidad de la cicatriz. Del total de desgarros encontramos un $27.7 \%$ de rerotura, porcentaje similar a series previamente publicadas.

Se describen los hallazgos del tendón operado y se correlacionan en algunos de ellos con exploración artroscópica demostrándose una buena sensibilidad del método. Consideramos a la ultrasonografía una alternativa válida para la evaluación post-quirúrgica del manguito rotador.

Palabras claves: Ultrasonido, Manguito rotador.

\section{Introducción}

Aproximadamente un $25 \%$ de los pacientes permanecen sintomáticos, después de la cirugía de manguito rotador ${ }^{(1-3)}$. Ello puede ser secundario a: Pinzamiento residual, rerotura, capsulitis adhesiva (hombro congelado), debilidad del músculo deltoides por desinserción o denervación y ausencia de bursa subacromiodeltoidea, la cual tarda 4-6 meses en formarse ${ }^{(3,5)}$.

Existen diversos métodos de imágenes para el estudio del estado del manguito rotador en post-operados como: artrografía, resonancia magnética y artroresonancia. El primero de ellos es de baja sensibilidad y especificidad ${ }^{(1,2,4,5)}$ y el segundo, no esta disponible para todos los pacientes en nuestro medio.

Yáñez $P$, Lúcia ME, Glasinovic A,Montenegro $S$. Ultrasonografía del manguito de los rotadores del hombro: Evaluación post-quirúrgica. Rev Chil Radiol 2002; 8:19-21. Correspondencia: Dra. Paulina Yáñez S. Clínica Santa María. Avenida Santa María 0410. Providencia. Santiago de Chile.E-mail: pyanez@chilesat.net
Hay pocos trabajos respecto a la evaluación del manguito rotador por ultrasonografía después de la cirugía. Una alta sensibilidad, especificidad y exactitud se ha publicado en algunos estudios ${ }^{(1,2)}$. Dado la importancia de detectar reroturas previo al desarrollo de una artropatía $^{(1)}$ se realizó éste estudio con el objetivo de evaluar la estabilidad de la cicatriz en forma dinámica y las características ecográficas del tendón operado.

\section{Material y método}

En forma prospectiva se exploró con ultrasonografía de alta resolución a 40 pacientes posterior a la reparación artroscópica de lesiones del manguito, realizadas entre los meses de Enero de 1999 a Enero del 2001. El promedio post quirúrgico fue de diez meses.

Se utilizó transductor de alta frecuencia de 10-12 $\mathrm{MHz}$,equipo ATL, HDI 3000. El estudio dinámico fue grabado en video. Se evaluó la indemnidad de los tendones del manguito rotador, explorando especialmente la "estabilidad de la cicatriz". Se define como estable, cuando se mueve en bloque y no se observa solución de continuidad o ausencia de tendón en relación a la tuberosidad mayor, dicho signo se ha descrito como confiable en estudios previos (Figura 1)(4).

El examen fue realizado por tres médicos radiólogos, con conocimiento previo del antecedente de reparación quirúrgica.

\section{Resultados}

La morfología y ecoestructura del manguito rotador post-quirúrgico cambia, apreciándose adelgazado, ecogénico y heterogéneo, con una mala definición de sus bordes, hay además algunos focos ecogénicos en relación a suturas e irregularidad de la cortical por anclas post-quirúrgicas ${ }^{(1,2,4,6,7)}$ (Figura 1).

De los 40 estudios realizados, posterior a la reparación artroscópica del manguito, se demostró: que de los 13 pacientes operados por rotura parcial (32.5\%) había estabilidad post-quirúrgica en 12 (92\%) al examen dinámico (Figura 2).

De los nueve pacientes operados $(22.5 \%)$ por roturas completas (no masivas), ocho (89\%) presentaban cicatriz estable al momento de la exploración (Figura 3).

En los 13 pacientes $(32.5 \%)$ con reparación de desgarros masivos, ocho (62\%) presentaba cicatriz estable, con movilización en bloque. 

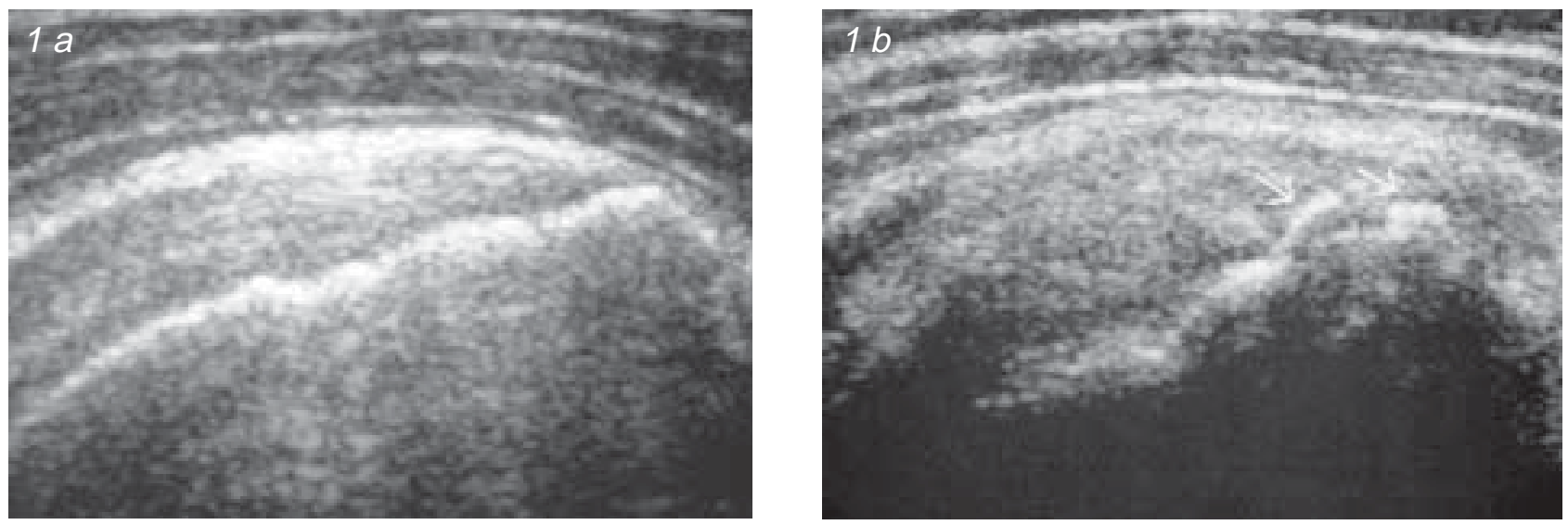

Figura 1 a,b. Cicatriz post quirúrgica estable. En diferentes posiciones hay movilidad en bloque y no se observa brecha o ausencia de tendón en relación a la tuberosidad mayor. El tendón es ecogénico y se observan focos ecogénicos gruesos (Flechas) correspondientes a anclas quirúrgicas.

Del total de pacientes siete presentaron reroturas (Figura 4). Los cinco restantes presentaron pinzamientos y hombro congelado.

Nuestros hallazgos se resumen en la Tabla I.

En cinco de nuestros pacientes diagnosticados como rerotura del tendón se efectuó una nueva exploración artroscópica confirmándose en todos ellos los hallazgos ultrasonográficos.

\section{Discusión}

Se describe que entre un $20 \%$ a $25 \%$ de los pacientes post-operados de manguito rotador, presentan dolor recurrente que puede ser explicado por diferentes causas tales como: Rerotura en un $20-30 \%$ según diferentes series ${ }^{(1-4)}$, capsulitis adhesiva, pinzamiento residual, tendinitis, etc. Esta variedad de causas dificulta la aproximación clínica ${ }^{(1-4)}$.

La principal complicación que se debe descartar es la rerotura del tendón operado(1).

Para evaluar el estado del manguito rotador en pacientes post-operados se han utilizado diversos métodos de imágenes, incluyendo la artrografía, ultrasonografía, resonancia magnética y artro-resonan$\mathrm{cia}^{(1-5)}$

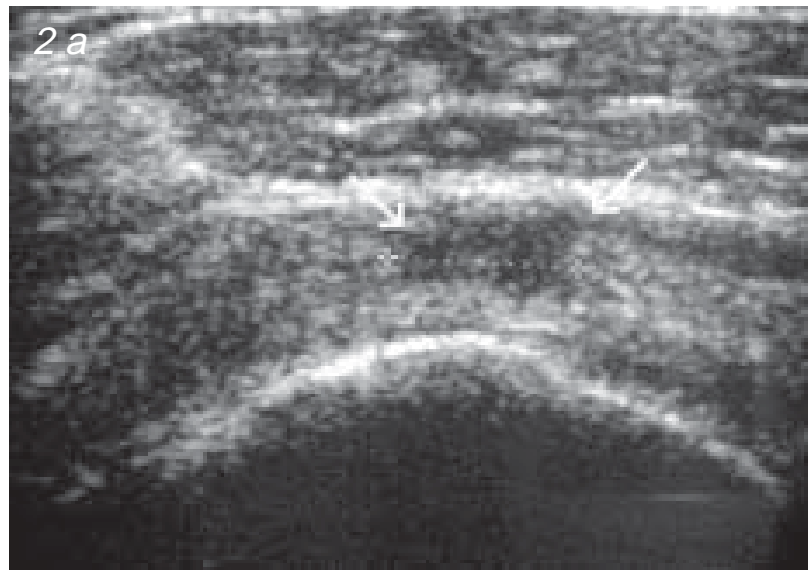

La artrografía presenta falsos negativos en el estudio de reroturas, debido a la presencia de adherencias y resección de la bursa subacromio-deltoidea ${ }^{(1,4)}$, obteniendo resultados pobres con sensibilidad de $60 \%$, especificidad de $50 \%$ y exactitud de $62,5 \%$.

Los estudios con resonancia magnética en búsqueda de reroturas muestran mejores resultados, describiéndose sensibilidad de $100 \%$ y especificidad de $97 \%$ para roturas totales ${ }^{(3)}$.

Algunos trabajos han utilizado ultrasonografía y han descrito buena correlación entre hallazgos ecográficos, clínicos, funcionales y post-quirúrgicos ${ }^{(1,2)}$.

Dado que en nuestro medio el acceso a resonancia se dificulta por su costo, y que la ultrasonografía músculo esquelética diagnóstica ha presentado un gran

\begin{tabular}{|c|c|c|c|}
\hline \multicolumn{4}{|c|}{$\begin{array}{l}\text { Tabla I: Lesión reparada v/s } \\
\text { hallazgos post-quirúrgicos }\end{array}$} \\
\hline & Estable & Retotura & $\begin{array}{c}\text { Total } 40 \\
\text { pacientes }\end{array}$ \\
\hline Desgarro parcial & $12(92 \%)$ & 1 (8\%) & 13 \\
\hline Desgarro completo & $8(89 \%)$ & $1(11 \%)$ & 9 \\
\hline Desgarro masivo & $8(62 \%)$ & $5(38 \%)$ & 13 \\
\hline Otros & - & - & 5 \\
\hline
\end{tabular}

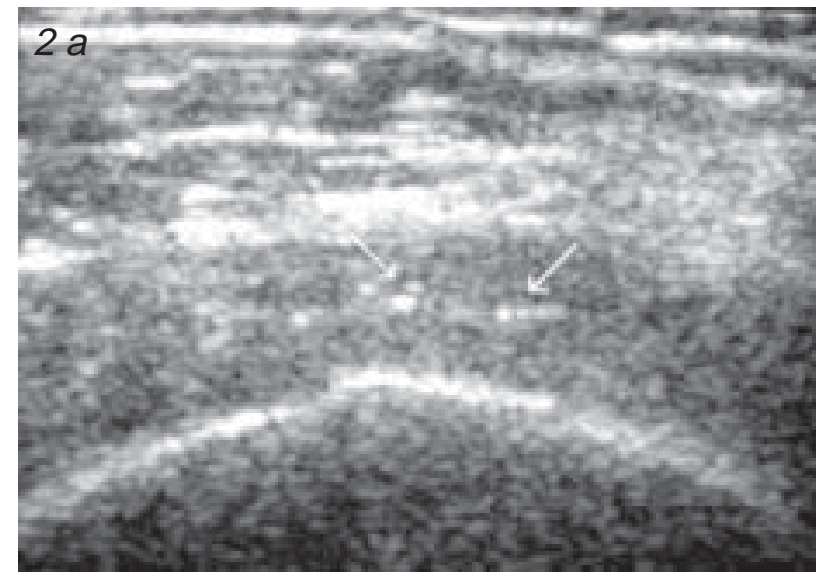

Figura 2 a,b. Tendón rotura parcial cicatrizada. a. Rotura (flechas) antes de cirugía. b. Post-cirugía, pequeñas áreas ecogénicas en el espesor del tendón (Flechas). 

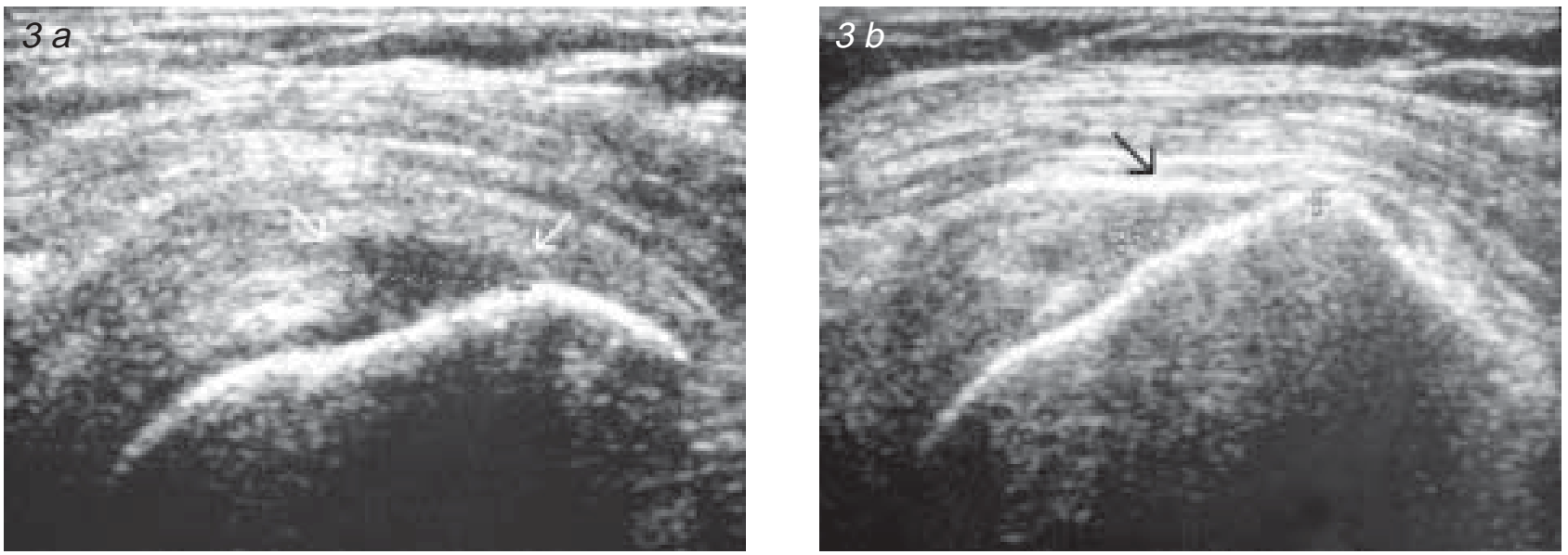

Figura 3 a,b. Tendón rotura completa cicatrizada. a. Rotura, antes de cirugía (Flechas). b. Cicatriz post-cirugía (flechas).

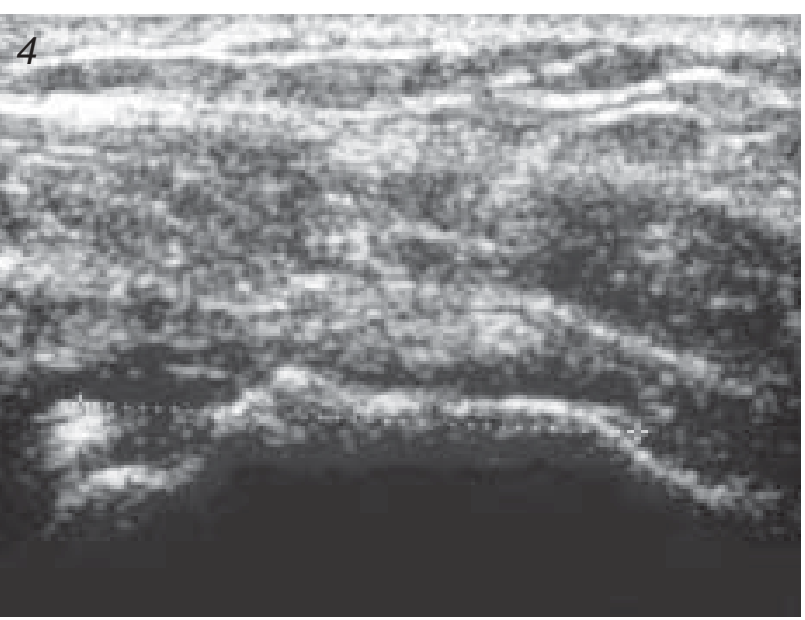

Figura 4. Rerotura del manguito rotador.

avance en el último período, se realizo éste estudio con el objeto de describir los hallazgos ecográficos de tendones post-operados.

En nuestra experiencia del total de desgarros completos (masivos y no masivos) reparados se observo un $27,7 \%$ de rerotura de la cicatriz, porcentaje similar al descrito por otros autores ${ }^{(1-4)}$.

Los hallazgos que hemos detectados son similares a los descrito en estudios previos ${ }^{(1,2,4,6,7)}$ y se caracterizan por:

a) Tendón de menor espesor.

b) Ecoestructura heterogénea, con pequeñas imágenes de mayor ecogenicidad en su espesor que pudiesen corresponder a material de sutura.

c) Inserción medial y posterior con irregularidad de la tuberosidad mayor logrando visualizar las anclas.

d) Perdida de la definición de los bordes del tendón, sin visualizar la bursa subacromiodeltoidea la cual es resecada en la cirugía ${ }^{(3-5)}$.

e) Movilización en bloque de la cicatriz. f) Cabeza humeral recubierta por cicatriz sin apreciar defecto o brecha en relación al tendón o falta de visualización.

Criterios de rotura parcial, completa y masiva ya han sido definidos en trabajos previos ${ }^{(1,6-8)}$.

Hay que tener presente las alteraciones previas para no sobrediagnosticar reroturas.

En trabajos publicados se ha descrito una sensibilidad de $100 \%$, especificidad de $90 \%$ y un $98 \%$ de exactitud con ultrasonografía ${ }^{(1)}$. En nuestra experiencia obtuvimos un $100 \%$ de correlación en los pacientes que se ha realizado una reexploración artroscópica. Para una mayor valoración del método, se deberá sin embargo, proseguir confirmando los hallazgos ultrasonográficos con la artroscopía.

\section{Bibliografía}

1. Van Holsbeeck M, I ntrocaso JH. Musculoskeletal Ultrasound. ED $2^{\circ}$ Mosby. Inc 2001. p : 463-516.

2. Knudsen HB, Gelineck J. Functional and Magnetic Resonance Image Evaluation After Simple Tendon Rotator Cuff Reconstruction. Journal of Shoulder and Elbow Surgery 1999; 8: 242-246.

3. Magee et al. MR Imaging of the Shoulder after Surgery. AJR 1997; 168: 925-928.

4. Crass et al. Sonography of the postoperative Rotator Cuff. AJR 1986; 146: 561-564.

5. Gusmen et al. MR Imaging of the Shoulder after rotator cuff repair. AJR 1997; 168: 559-563.

6. Rethy $\mathrm{CH}$, Etienne C. Guidelines and Gamuts in Musculoskeletal Ultrasound. ED by Willy-Liss. INC 1999; p. 39-72.

7. Bouffard JA, Lee SM. Ultrasonography of the Shoulder. Seminars in ultrasound CT, and MRI 2000; 21: 164-191.

8. Yáñez P, Lúcia M, Glasinovic A, Montenegro S. Correlación ultrasonográfica vs artroscopía en rotura del manguito rotador. Rev Chil Radiol 2001; 7: 117-119. 